\title{
Performance of shear-waves elastography in the non-invasive assessment of thyroid stiffness in patients with viral hepatitis
}

\author{
Oana Săndulescu ${ }^{1,2^{*}}$, Anca Streinu-Cercel ${ }^{1,2}$, Monica Andreea Stoica ${ }^{2}$, Daniela Manolache ${ }^{2}$, Adrian Streinu-Cercel ${ }^{1,2}$ \\ From The 10th Edition of the Scientific Days of the National Institute for Infectious Diseases "Prof Dr Matei \\ Bals" \\ Bucharest, Romania. 15-17 October 2014
}

\section{Background}

In chronic HCV infection, high rates of viral replication are often associated with progressive liver fibrosis. As HCV has also been shown to replicate in other organs, such as the thyroid [1], we have performed a pilot study to assess thyroid stiffness through non-invasive elastography.

\section{Methods}

One trained operator performed shear-waves elastography (SWE) of the liver and thyroid in patients with chronic $\mathrm{HCV}$ infection, using Aixplorer (SuperSonic Imagine, Aix-en-Provence, France).

\section{Results}

We assessed 21 patients with chronic HCV infection, with a male-to-female ratio of $0.6: 1$. The mean age was $51.4 \pm 11.9$ years. The mean duration of HCV infection was $5.2 \pm 5.5$ years (range: $0-20$ years). The predominant HCV genotype was $1 \mathrm{~b}$ (in 16 patients - data not available for 5 patients), and the predominant IL28-B genotype was CT (9 patients), followed by TT (7 patients) and CC (3 patients) - IL28-B data were not available for 2 patients.

Most $(17,81.0 \%)$ of the patients had received prior anti-HCV therapy with peg-interferon+ribavirin (PR) or direct-acting antivirals (DAA)-based treatment, and 13 of them displayed SVR (12 with DAA-based therapy and one with PR - the patient had an IL28-B genotype CC), while 4 displayed non-response to PR (IL28-B genotypes CT and TT).

Eight of the patients (38.1\%) had a previously diagnosed thyroid dysfunction, and 4 of them (19.0\%) were under

\footnotetext{
* Correspondence: oanasandulescu1@gmail.com

${ }^{1}$ Carol Davila University of Medicine and Pharmacy, Bucharest, Romania
}

Full list of author information is available at the end of the article thyroid substitution treatment at the time of evaluation. Five of the patients $(23.8 \%)$ presented thyroid nodules on ultrasound examination.

The mean liver SWE was $9.6 \pm 4.2 \mathrm{kPa}$ and the mean thyroid SWE was $25.1 \pm 10.4 \mathrm{kPa}$ overall, and $26.2 \pm$ $11.3 \mathrm{kPa}$ for the left thyroid lobe and $24.7 \pm 13.0 \mathrm{kPa}$ for the right thyroid lobe.

\section{Conclusion}

This pilot study warrants further dynamic assessment of liver and thyroid stiffness in patients with chronic $\mathrm{HCV}$ infection, on larger study groups. To our knowledge, this is the first such study on thyroid stiffness in HCV-infected patients.

\section{Acknowledgement \\ This paper is partially supported by the Sectoral Operational Programme Human Resources Development (SOP HRD), financed from the European Social Fund and by the Romanian Government under the contract numbers POSDRU/159/1.5/S/137390.}

\section{Authors' details \\ ${ }^{1}$ Carol Davila University of Medicine and Pharmacy, Bucharest, Romania. ${ }^{2}$ National Institute for Infectious Diseases "Prof. Dr. Matei Balş", Bucharest, Romania.}

\section{Published: 15 October 2014}

\section{References}

1. Bartolomé J, Rodríguez-Iñigo E, Quadros P, Vidal S, Pascual-Miguelañez I, Rodríguez-Montes JA, García-Sancho L, Carreño V: Detection of hepatitis C virus in thyroid tissue from patients with chronic HCV infection. $J$ Med Virol 2008, 80:1588-94.

2. Streinu-Cercel A: Hepatitis C in the interferon-free era. GERMS 2013, 3:114.

doi:10.1186/1471-2334-14-S7-P54

Cite this article as: Săndulescu et al:: Performance of shear-waves elastography in the non-invasive assessment of thyroid stiffness in patients with viral hepatitis. BMC Infectious Diseases 2014 14(Suppl 7):P54. 